\title{
Talbot effect in metallic gratings under Gaussian illumination
}

\author{
Luis Miguel Sanchez-Brea *, Francisco Jose Torcal-Milla, Eusebio Bernabeu \\ Universidad Complutense de Madrid, Optics Department, Applied Optics Complutense Group, Facultad de Ciencias Físicas, \\ Ciudad Universitaria s.n., 28040 Madrid, Spain
}

Received 23 February 2007; received in revised form 17 May 2007; accepted 25 May 2007

\begin{abstract}
Metallic gratings can be found in applications such as optical metrology. Due to their fabrication process, the surface presents a certain roughness. In this work, the effect of roughness on Talbot effect has been analyzed when the grating is illuminated with a Gaussian beam. A model based on Fresnel regime is used in order to determine the intensity distribution in the near field. Contrast of the selfimages is obtained and it is found that it decreases in terms of the distance between the grating and the observation plane. When the autocorrelation function of roughness presents a Gaussian behaviour, the diffracted beams are still Gaussian although some of their properties change. For example, the width of the diffracted beams increases with respect to the case of the standard chrome on glass gratings. On the other hand, the power of each diffracted beam is independent on the roughness properties of the surface.

(c) 2007 Elsevier B.V. All rights reserved.
\end{abstract}

PACS: 42.25.-p; 42.25.Fx; 42.25.Hz; 42.79.Dj

\section{Introduction}

In most cases, diffraction gratings made of glass or chrome on a glass substrate are used. For these gratings, the current fabrication process allows periods ranging from microns to several nanometers. However, there exist cases, such as optical metrology, where very long diffraction gratings $(>3 \mathrm{~m})$ are required. In these cases, glass gratings are not available since they are very difficult to manufacture and handle. Then, diffraction gratings engraved in a steel tape substrate are used. Up to date, the period of such gratings can only be found in the hundred of microns range. The surface of these diffraction gratings presents a certain roughness. Recently steel tape gratings with periods around $20-40 \mu \mathrm{m}$ have been developed and then the diffractive behaviour of such gratings becomes important. For example, Talbot effect becomes relevant. In Talbot effect, when a periodic object is illuminated with a monochromatic light wave, images of the object appear at certain

\footnotetext{
${ }^{*}$ Corresponding author.

E-mail address: sanchezbrea@fis.ucm.es (L.M. Sanchez-Brea).
}

distances behind it [1-3]. This phenomenon has received continuous attention, not only from a theoretical point of view, but also because of its wide range of applications, such as spectroscopy, optical metrology, moiré interferometry, laser array illumination, phase locking of the laser array, etc. [4-7]. The case of gratings without roughness, illuminated with a Gaussian beam has been analyzed in the classical work by Szapiel and Patorski [8].

In this work, the effect of roughness of metallic reflection gratings on the self-imaging process is analyzed when they are illuminated with a Gaussian beam. Roughness is modelled using statistical techniques in order to determine the mutual intensity function, and then the intensity distribution at the observation plane.

\section{Theoretical approach}

Let us consider the scheme of Fig. 1. A diffraction grating with a rough surface is illuminated with a Gaussian beam. We assume that the rough diffraction grating can be mathematically defined as the multiplication of two factors 


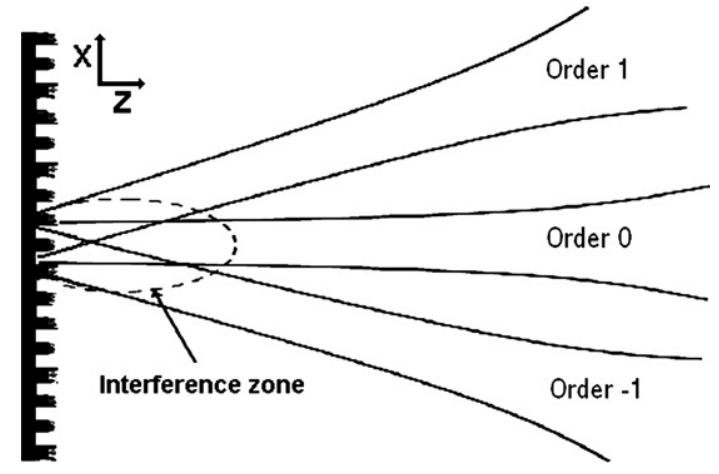

Fig. 1. Scheme that shows the propagation of the diffraction orders in the near field produced by a grating with roughness.

$G(x)=g(x) t(x)$,

where $x$ is the transversal coordinate at the grating plane. The first factor corresponds to a grating described as

$g(x)=\sum_{n} a_{n} \exp [\operatorname{iq} n(x+\Delta x)]$,

being $a_{n}$ the Fourier coefficients of the grating, $q=2 \pi / p, p$ the grating period and $\Delta x$ a relative displacement of the grating. The second factor is the reflectance of the surface and it is related to the surface topography $\zeta\left(x_{1}\right)$, which is random and whose average level is null, $\left\langle\zeta\left(x_{1}\right)\right\rangle=0$. We will assume an infinite conductivity. We will also consider a normal distribution in heights $w(z)=\exp \left(-z^{2} / 2 \sigma^{2}\right) /$ $\sqrt{2 \pi} \sigma$ where $z=\zeta\left(x_{1}\right)$ and $\sigma$ is the standard deviation [9]. Then, the reflectance results $t\left(x_{1}\right)=\exp \left[\mathrm{i} 2 k \zeta\left(x_{1}\right)\right]$, where $k=2 \pi / \lambda$ and $\lambda$ is the wavelength. When a finite conductivity needs to be considered, this reflectance can be obtained considering in addition the Fresnel equations for reflectance [9]. The correlation properties of roughness will be defined below in relation to the mutual intensity function.

Let us consider that the diffraction grating is illuminated with a monochromatic Gaussian beam. The period of the grating $p$ is assumed much larger than the wavelength $\lambda$ of the incident field, thus a scalar treatment is possible. For simplicity, the waist of the Gaussian beam is placed at the grating plane

$U_{0}\left(x_{0}\right)=A_{0} \exp \left[-\left(\frac{x_{0}}{\omega_{0}}\right)^{2}\right]$,

where $A_{0}$ is the maximum amplitude and $\omega_{0}$ is the beam width. Then, the amplitude just after the grating is

$U_{1}\left(x_{1}\right)=A_{0} t\left(x_{1}\right) \exp \left[-\left(\frac{x_{1}}{\omega_{0}}\right)^{2}\right] \sum_{n} a_{n} \exp \left(\mathrm{i} q n x_{1}\right)$,

where we have considered, without loss of generality, that $\Delta x=0$. Due to roughness, statistical properties of the field need to be considered. In particular, the mutual intensity of the beam just after the grating is [10]

$J\left(x_{1}, x_{1}^{\prime}\right)=\left\langle U_{1}\left(x_{1}\right) U_{1}^{*}\left(x_{1}^{\prime}\right)\right\rangle$, where $\langle\cdot\rangle$ means averaging. Normally, the topography $\zeta\left(x_{1}\right)$ is unknown. However, the statistical properties of the surface are included in the autocorrelation function $\left\langle t\left(x_{1}\right) t^{*}\left(x_{1}^{\prime}\right)\right\rangle$ which is measurable. There exist several common models to fit the experimental autocorrelation function [11-13]. Considering several theoretical and experimental works $[14,15]$, we have used a Gaussian autocorrelation function

$\left\langle t\left(x_{1}\right) t^{*}\left(x_{1}^{\prime}\right)\right\rangle=\exp \left[-\frac{\left(x_{1}-x_{1}^{\prime}\right)^{2}}{T_{0}^{2}}\right]$,

where $T_{0}$ is the correlation length of the field [9]. Then the mutual intensity just after the grating, given by Eq. (5), results in

$J\left(x_{1}, x_{1}^{\prime}\right)=\left|A_{0}\right|^{2} \mathrm{e}^{-\left(\frac{x_{1}-x_{1}^{\prime}}{T_{0}}\right)^{2}} \mathrm{e}^{-\left(\frac{x_{1}+x_{1}^{\prime}}{\omega_{0}}\right)^{2}} \sum_{n} \sum_{n^{\prime}} a_{n} a_{n^{\prime}}^{*} \mathrm{e}^{\mathrm{i} q\left(n x_{1}-n^{\prime} x_{1}^{\prime}\right)}$.

We are interested in the near field. Then, the mutual intensity at a distance $z$ is obtained performing the Fresnel propagation of the mutual intensity at $z=0[10]$

$$
\begin{aligned}
J\left(x_{2}, x_{2}^{\prime}\right)= & \int_{-\infty}^{\infty} \int_{-\infty}^{\infty} J\left(x_{1}, x_{1}^{\prime}\right) \\
& \times \exp \left[\frac{\mathrm{i} k}{2 z}\left(x_{2}-x_{1}\right)^{2}\right] \exp \left[-\frac{\mathrm{i} k}{2 z}\left(x_{2}^{\prime}-x_{1}^{\prime}\right)^{2}\right] \mathrm{d} x_{1} \mathrm{~d} x_{1}^{\prime} .
\end{aligned}
$$

The average intensity is obtained directly from the mutual intensity using $\left\langle I\left(x_{2}\right)\right\rangle=J\left(x_{2}, x_{2}\right)$. Introducing the mutual intensity function obtained in Eq. (7) into Eq. (8), and solving the integrals, the average intensity distribution at the observation plane results in

$$
\begin{aligned}
\left\langle I_{2}\left(x_{2}, z\right)\right\rangle \propto & \sum_{n} \sum_{n^{\prime}} a_{n^{\prime}}^{*} a_{n} \exp \left[\frac{\mathrm{i}\left(n-n^{\prime}\right) q x_{2}}{1+\left(z / z_{0}\right)^{2}\left(1+\omega_{0}^{2} / T_{0}^{2}\right)}\right] \\
& \times \exp \left[\frac{-2\left[x_{2}-\left(n+n^{\prime}\right) q z / 2 k\right]^{2}}{\omega^{2}(z)+2\left(\lambda z / \pi T_{0}^{2}\right)}\right] \\
& \times \exp \left[\frac{-\mathrm{i} z\left(n^{2}-n^{\prime 2}\right) q^{2} / 2 k}{1+\left(z / z_{0}\right)^{2}\left(1+\omega_{0}^{2} / T_{0}^{2}\right)}\right] \\
& \times \exp \left[\frac{-\left(n-n^{\prime}\right)^{2} q^{2} \omega_{0}^{2} / 8}{1+\left(z_{0} / z\right)^{2} /\left(1+2 \omega_{0}^{2} / T_{0}^{2}\right)}\right],
\end{aligned}
$$

where $\omega^{2}(z)=\omega_{0}^{2}\left[1+\left(z / z_{0}\right)^{2}\right]$ and $z_{0}=\pi \omega_{0}^{2} / \lambda$ is the Rayleigh distance [16]. The first exponential factor of Eq. (9) accounts for the period of the fringes which results $\hat{p}=p\left[1+\left(z / z_{0}\right)^{2}\left(1+\omega_{0}^{2} / T_{0}^{2}\right)\right]$. In principle, $\hat{p}$ depends on roughness. However, this dependence is not normally significant since it is notorious when $z \gg z_{0}$, and in this regime the intensity goes to zero due to the exponential diminishing factors of Eq. (9). The second exponential factor accounts for the direction of propagation of the different diffraction orders and their profiles. It can be observed that 
the profile of the diffraction orders is also Gaussian. However, the width of the diffracted beams $\omega_{\text {Rough }}^{2}=\omega^{2}(z)+$ $2\left(\lambda z / \pi T_{0}\right)^{2}$ increases when the correlation length $T_{0}$ decreases. The third exponential factor accounts for the Talbot effect and shows the positions where Talbot selfimages are formed. Finally, the fourth exponential factor accounts of an interaction between different orders of diffraction.

When the experimental autocorrelation function of the topography fits better to another model (exponential, triangular, etc.), the integrals of Eq. (8) normally need to be solved by numerical methods.

In order to understand the self-imaging process under Gaussian illumination in presence of roughness, several computations have been performed. For this, we have assumed that a metallic grating can be modelled as a binary phase grating. Then, the Fourier coefficients of the grating are

$$
a_{n}= \begin{cases}\alpha\left(\mathrm{e}^{-\mathrm{i} \delta}-1\right) \operatorname{sinc}(n \pi \alpha), & n \neq 0 \\ \alpha\left(\mathrm{e}^{-\mathrm{i} \delta}-1\right)+1, & n=0\end{cases}
$$

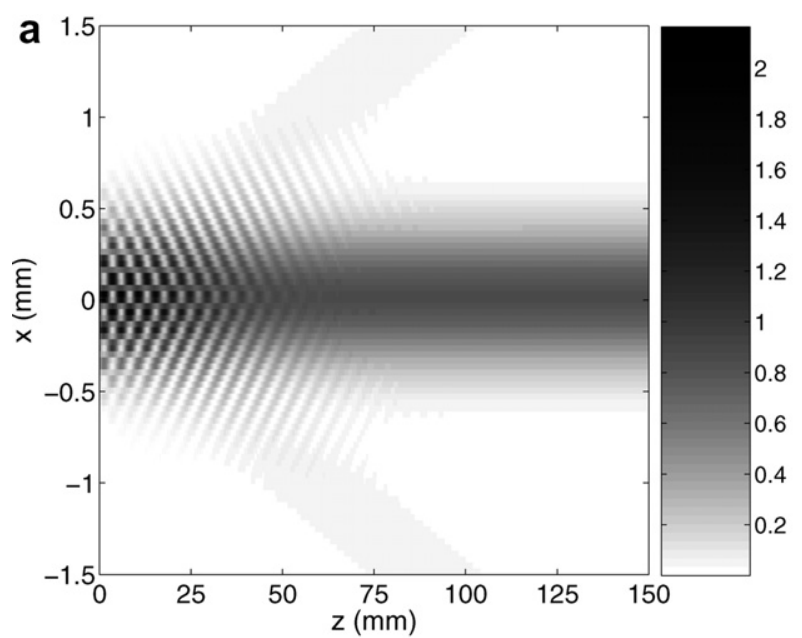

where $\alpha$ is the ratio between the upper and the lower part of the grating and $\delta$ is the phase delay produced by the grating.

In Fig. 2, it is shown that the zone of interference between the different diffraction orders decreases when roughness is considered.

For the limit case of null roughness $\left(T_{0} \rightarrow \infty\right)$ the intensity results in

$$
\begin{aligned}
\left\langle I_{2}\left(x_{2}, z\right)\right\rangle \propto & \sum_{n} \sum_{n^{\prime}} a_{n^{\prime}}^{*} a_{n} \exp \left[\frac{\mathrm{i}\left(n-n^{\prime}\right) q x_{2}}{1+\left(z / z_{0}\right)^{2}}\right] \\
& \times \exp \left[\frac{-2\left[x_{2}-\left(n+n^{\prime}\right) q z / 2 k\right]^{2}}{\omega^{2}(z)}\right] \\
& \times \exp \left[\frac{-\mathrm{i} z\left(n^{2}-n^{\prime 2}\right) q^{2} / 2 k}{1+\left(z / z_{0}\right)^{2}}\right] \\
& \times \exp \left[\frac{-\left(n-n^{\prime}\right)^{2} q^{2} \omega_{0}^{2} / 8}{1+\left(z_{0} / z\right)^{2}}\right] .
\end{aligned}
$$

Finally, when we are in a plane wave illumination approach, $\omega_{0} \rightarrow \infty$, the classical result for Talbot effect is recovered [1] as

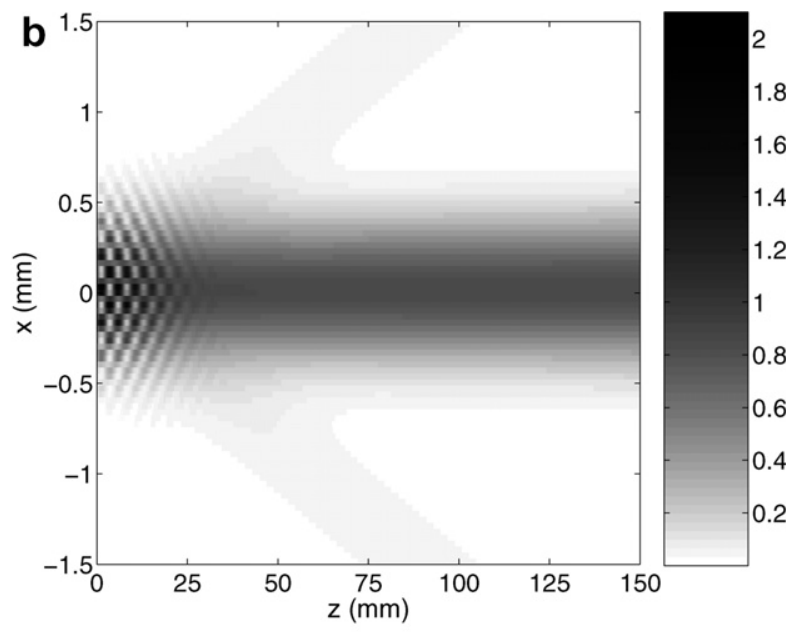

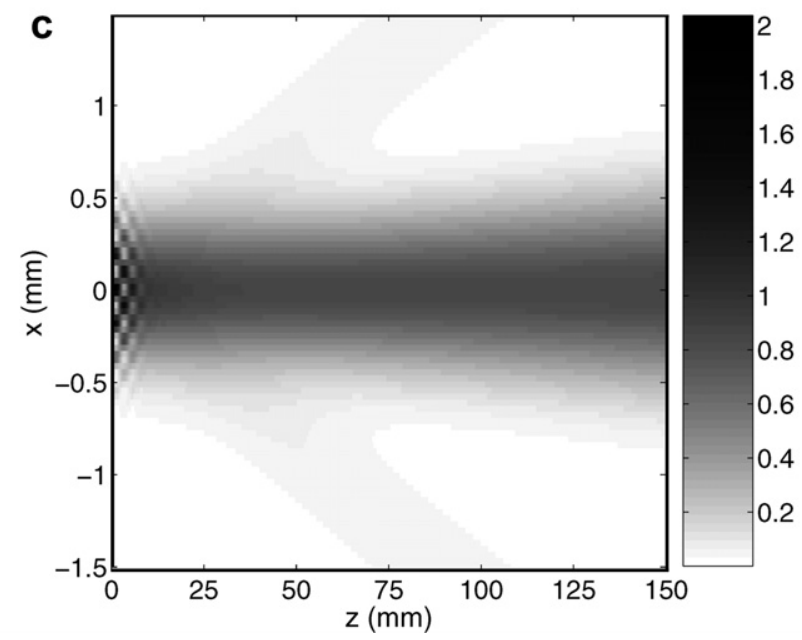

Fig. 2. Talbot planes. The period of the grating is $p=40 \mu \mathrm{m}$, the wavelength of the incident beam is $\lambda=0.68 \mu \mathrm{m}$, the width at the grating is $\omega_{0}=500 \mu \mathrm{m}$ : (a) without roughness, $T_{0} \rightarrow \infty$, (b) with high roughness, $T_{0}=300 \mu \mathrm{m}$ and (c) with very high roughness, $T_{0}=100 \mu \mathrm{m}$. In this example, we have considered a phase grating and the summatories runs from $n, n^{\prime}=-11: 11$. Note that aliasing is produced in the figures since the period of the grating is $p=40 \mu \mathrm{m}$ and the size of $x$ in the figures is $3000 \mu \mathrm{m}$. 


$$
\begin{aligned}
\left\langle I_{2}\left(x_{2}, z\right)\right\rangle \propto & \sum_{n} \sum_{n^{\prime}} a_{n^{\prime}}^{*} a_{n} \\
& \times \exp \left[\mathrm{i}\left(n-n^{\prime}\right) q x_{2}\right] \exp \left[\mathrm{i}\left(n^{\prime 2}-n^{2}\right) \frac{q^{2}}{2 k} z\right] .
\end{aligned}
$$

To characterize the quality of the self-images produced by the steel tape grating, an important parameter is the contrast, which is defined as

$C=\frac{I_{\mathrm{MAX}}-I_{\mathrm{MIN}}}{I_{\mathrm{MAX}}+I_{\mathrm{MIN}}}$,

where $I_{\mathrm{MAX}}$ and $I_{\mathrm{MIN}}$ are the maximum and minimum intensity of the fringes, respectively. Since the incident beam is Gaussian, the intensity depends on the location $x_{2}$ of the beam with respect of the origin of coordinates. A solution is to determine the contrast at $x_{2}=0$, where the intensity of the incident beam presents a maximum.
Then, we define the maximum and minimum intensity as those obtained at $\Delta x=0$ and $\Delta x=p / 2$, where $\Delta x$ is a relative displacement of the grating as defined in Eq. (2). Then, contrast results in

$C=\frac{\sum_{n=\infty}^{\infty} \sum_{n^{\prime}=\infty}^{\infty} \Gamma_{n, n^{\prime}}(z) a_{n} a_{n^{\prime}}^{*}\left\{1-\exp \left[\mathrm{i} \pi\left(n-n^{\prime}\right)\right]\right\}}{\sum_{n=\infty}^{\infty} \sum_{n^{\prime}=\infty}^{\infty} \Gamma_{n, n^{\prime}}(z) a_{n} a_{n^{\prime}}^{*}\left\{1+\exp \left[\mathrm{i} \pi\left(n-n^{\prime}\right)\right]\right\}}$,

where

$$
\begin{aligned}
& \Gamma=\exp \left[\alpha\left(n^{2}-n^{\prime 2}\right)+\beta\left(n+n^{\prime}\right)^{2}+\gamma\left(n-n^{\prime}\right)^{2}\right], \\
& \alpha=\frac{-\mathrm{i} q^{2} z}{\left\{2 k\left[1+\left(\frac{\lambda z}{\pi \omega_{0}^{2}}\right)^{2}\left(1+\frac{\omega_{0}^{2}}{T_{0}^{2}}\right)\right]\right\}}, \\
& \beta=\frac{-q^{2} z^{2}}{2 k^{2}\left\{2\left(\frac{\lambda z}{\pi T_{0}}\right)^{2}+\left[1+\left(\frac{\lambda z}{\pi \omega_{0}^{2}}\right)^{2}\right] \omega_{0}^{2}\right\}},
\end{aligned}
$$
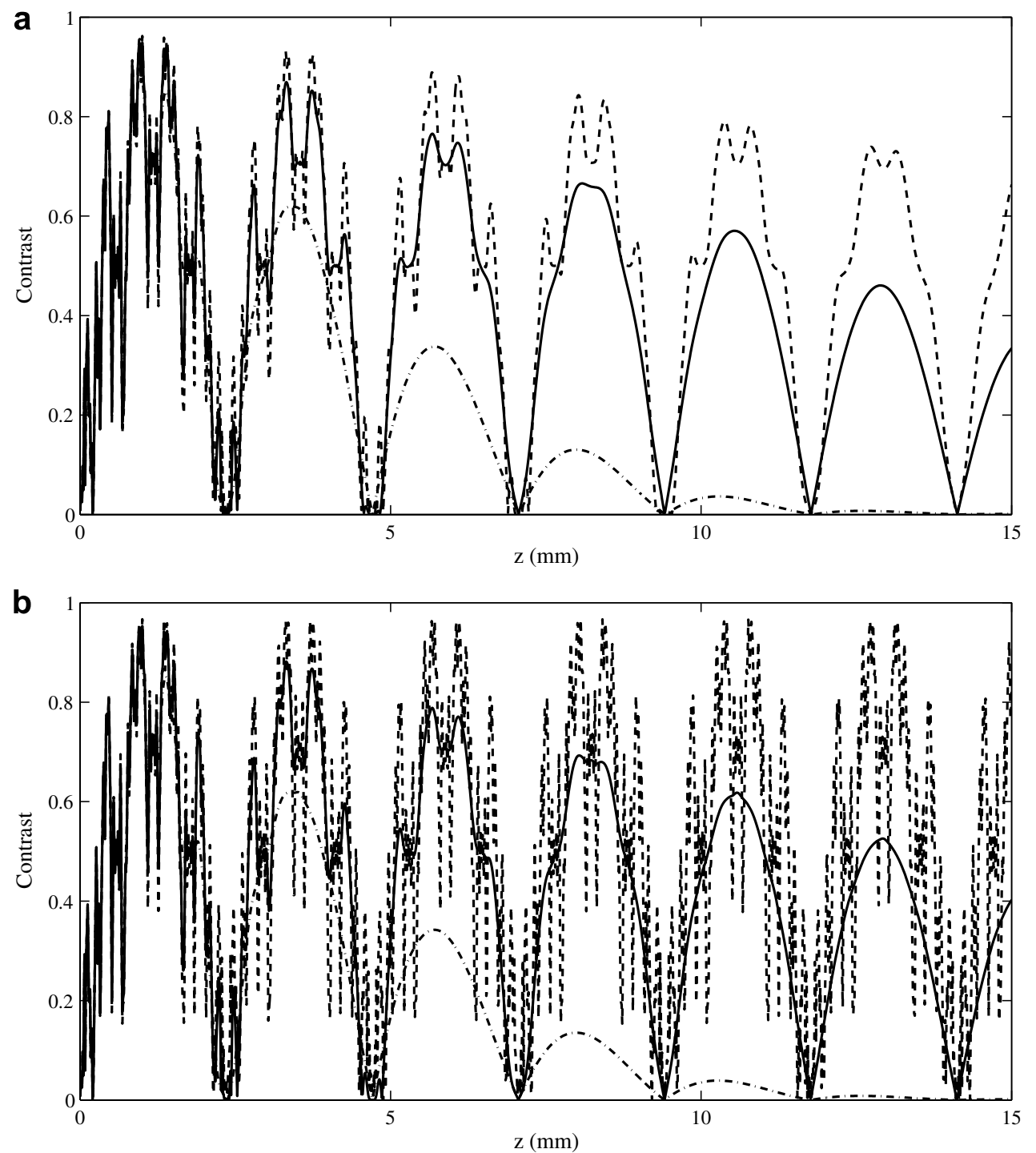

Fig. 3. Contrast in terms of the distance $z$ from the grating to the observation plane $p=40 \mu \mathrm{m}, \lambda=0.68 \mu \mathrm{m}$ for several roughness parameters: $T_{0} \rightarrow \infty$, low roughness (dash), $T_{0}=300 \mu \mathrm{m}$, high roughness (solid), and $T_{0}=100 \mu \mathrm{m}$, very high roughness (dash + dot): (a) with Gaussian illumination, beam waist $\omega_{0}=500 \mu \mathrm{m}$ and (b) with collimated illumination, $\omega_{0} \rightarrow \infty$. In this example, we have considered a phase grating and the summatories runs from $n, n^{\prime}=-11: 11$ 


$$
\gamma=\frac{-q^{2} \omega_{0}^{2}}{8\left[1+\frac{\pi^{2} \omega_{0}^{4}}{(\lambda z)^{2}\left(1+2 \frac{\omega_{0}^{2}}{T_{0}^{2}}\right)}\right]} .
$$

In Fig. 3a, the contrast is shown for different roughness parameters and a finite beam width $\left(\omega_{0}=500 \mu \mathrm{m}\right)$. Comparing this result to that obtained for the case of plane wave illumination $\left(\omega_{0} \rightarrow \infty\right)$, Fig. $3 b$, it is shown that roughness affects to contrast. It decreases faster when roughness is present. We can also observe that when high roughness is present only the first Talbot planes appear and eventually Talbot planes disappear for very high roughness.

When another model of autocorrelation function is used, the behaviour of the contrast is similar to that obtained in Fig. 3. Roughness produces a decrease of the intensity in terms of the distance between the grating and the observation plane, being the envelope different for different models.

\section{Non-interference zone}

As it is shown in Fig. 2, there is a distance from which the different diffraction orders do not interfere. This distance corresponds to $z_{N}=p \omega_{0} / 2 \lambda$, which can be easily obtained from a geometrical analysis. For distances longer than $z_{N}$, Talbot planes disappear since the different diffraction orders do not overlap. In this regime, $z>z_{N}$ the intensity results in

$$
\left\langle I_{2}\left(x_{2}, z\right)\right\rangle \propto \sum_{n}\left|a_{n}\right|^{2} \exp \left\{\frac{-2 z^{2}(\theta-n \lambda / p)^{2}}{\omega^{2}(z)+2\left(\lambda z / \pi T_{0}\right)^{2}}\right\},
$$

where $\theta=x_{2} / z$. As it is well known, several diffraction beams appear. The maxima of the diffracted Gaussian beams are placed at $p \theta=n \lambda$, which is the linear version of the well-known grating equation. It is observed that, when the autocorrelation function of roughness is Gaussian, Eq. (6), the diffracted beams are also Gaussian. The width of the Gaussian beams is affected by roughness, increasing from $\omega^{2}(z)$ to $\omega^{2}(z)+2\left(\lambda z / \pi T_{0}\right)^{2}$, that is, the shorter the correlation length of roughness, the wider the diffracted beams. This increasing can be observed in Fig. 2 .

It is also important to analyze whether roughness produces a redistribution of the power amongst diffraction orders. Power is defined as the total intensity for each order $n$, which results [16]

$$
\begin{aligned}
P_{n} & =\frac{1}{\sqrt{1+\left(\frac{z}{z_{0}}\right)^{2}\left[1+\left(\frac{\omega_{0}}{T_{0}}\right)^{2}\right]}} \int_{-\infty}^{\infty}\left|a_{n}\right|^{2} \exp \left\{\frac{-2\left(x_{2}-n \lambda z / p\right)^{2}}{\omega^{2}(z)+2\left(\lambda z / \pi T_{0}\right)^{2}}\right\} \mathrm{d} x_{2} \\
& =\sqrt{\frac{\pi}{2} \omega_{0}\left|a_{n}\right|^{2},}
\end{aligned}
$$

which is independent on the correlation length of the field. As a consequence, there is not a redistribution of power amongst the diffraction orders due to roughness.

\section{Conclusions}

In this work, we have analyzed Talbot effect for the case of metallic gratings when they are illuminated with a Gaussian beam. An example of this kind of gratings is those engraved on a steel substrate. These gratings are not ideal since they present a rough surface. When the autocorrelation function of roughness presents a Gaussian behaviour, then the diffracted beams are still Gaussian, but their parameters change. The width of these beams increases with respect those obtained with ideal gratings. Also, at locations where the diffraction orders interfere, a self-imaging process happens, but the contrast of fringes is smaller than for the case where roughness is not present. On the other, hand power redistribution amongst the different orders of diffraction does not happen.

\section{Acknowledgements}

This work has been supported by the DPI2005-02860 project of the Ministerio de Educación y Ciencia of Spain and the "Tecnologías en ecología, alta precisión y productividad, multifuncionalidad, y tecnologías de la información y comunicaciones en Máquina Herramienta" CENIT project of the Ministerio de Industria, turismo y comercio. Sanchez-Brea is currently contracted by the Universidad Complutense de Madrid under the "Ramón y Cajal" research program of the Ministerio de Educación y Ciencia of Spain.

\section{References}

[1] W.H.F. Talbot, Philos. Mag. 9 (1836) 401.

[2] K. Patorski, Prog. Optics 27 (1989) 1.

[3] E. Keren, O. Kafri, J. Opt. Soc. Am. A 2 (2) (1985) 111.

[4] A.W. Lohmann, D.E. Silva, Opt. Commun. 2 (1971) 413.

[5] G. Schirripa Spagnolo, D. Ambrosini, D. Paoletti, J. Opt. A: Pure Appl. Opt. 4 (2002) S376.

[6] B.F. Oreb, R.G. Dorsch, Appl. Opt. 33 (1994) 7955.

[7] S. Wei, S. Wu, I. Kao, F.P. Chiang, Trans. ASME J. Electron. Packag. 120 (1998) 166

[8] S. Szapiel, K. Patorski, Opt. Acta 26 (4) (1979) 439.

[9] P. Beckmann, A. Spizzichino, The Scattering of Electromagnetic Waves from Rough Surfaces, Artech House, 1987.

[10] J.W. Goodman, Statistical Optics, John Wiley \& Sons, New York, 1985.

[11] J.C. Dainty, Laser Speckle and Related Phenomena, Springer-Verlag, 1984.

[12] J.A. Ogilvy, Theory of Wave Scattering from Random Rough Surfaces, IOP, 1991.

[13] F. Perez-Quintian, A. Lutenberg, M.A. Rebollo, Appl. Opt. 45 (20) (2006) 4821

[14] V.Ya. Mendeleev, S.N. Skovorod'ko, Optics and Spectroscopy 94 (3) (2003) 437.

[15] V. Celli, A.A. Maradudin, A.M. Marvin, A.R. McGurn, J. Opt. Soc. Am. A 2 (12) (1985) 2225

[16] B.E.A. Saleh, M.C. Teich, Fundamentals of Photonics, John Wiley \& Sons, 1991. 